\title{
RESPUESTA DEL CULTIVO DE ALCACHOFA MULTIPLICADA POR SEMILLA A DIFERENTES DOSIS DE RIEGO
}

\author{
Baixauli, $C^{(1)}$.,Aguilar, J.M ${ }^{(1)}$., Giner, $A^{(1)}$.,Nájera, $I^{(1)}$.,Pomares, $F^{(2)}$., Tarazona, $F^{(2)}$,Estela, $M^{(2)}$ \\ ${ }^{1}$ Investigador. Fundación Cajamar Comunidad Valenciana. Camino del Cementerio nuevo \\ s/n. 46200 Paiporta (Valencia). carlosbaixauli@cajamar.com \\ 2 Investigador. Instituto Valenciano de Investigaciones Agrarias. Carretera Moncada- \\ Náquera, km 4.5. 46113 Moncada (Valencia).pomares_fer@gva.es
}

\section{Resumen}

El objetivo de la experiencia fue evaluar los efectos de tres dosis de riego en diferentes parámetros productivos y cualitativos de una variedad de alcachofa multiplicada por semilla en riego por goteo.

El ensayo se realizó en el Centro de Cajamar en Paiporta (Valencia). La plantación de alcachofa de semilla, cv. Symphony $F_{1}$ (Nunhems), se efectuó el 22/7/2010, con un marco de plantación de $1.67 \times 0.78 \mathrm{~m}\left(0.77 \mathrm{pl} \mathrm{m}^{-2}\right)$. Las plantas se sometieron a tres aplicaciones con ácido giberélico a 60 ppm en las fechas: 6/9/10, 21/9/10 y 6/10/10, respectivamente. La recolección se realizó desde el 4/11/10 al 17/5/11. Las dosis de riego aplicadas se calcularon en base a la $\mathrm{ET}_{\mathrm{c}}$, a partir de la evapotranspiración de referencia $\left(\mathrm{ET}_{\mathrm{o}}\right)$, obtenida siguiendo el método Penman-Monteith y los coeficientes de cultivo $\left(\mathrm{K}_{\mathrm{c}}\right)$, con valores de 0.35 , 1.20 y 1.05 , durante los periodos inicial, intermedio y final, respectivamente. Los volúmenes de agua aplicados fueron: $2.943,4.305$ y $6.031 \mathrm{~m}^{3}$ ha-1 , en los tratamientos $R_{1}, R_{2}, R_{3}$, respectivamente. La lluvia contabilizada durante el ciclo del cultivo fue de $374 \mathrm{~mm}$. Estas dosis de riego resultan equivalentes al 68,100 y $140 \%$ de la $\mathrm{ET}_{\mathrm{c}}$, para las distintas dosis analizadas.

Se aplicó el mismo abonado en las tres dosis de riego a base de $166 \mathrm{~kg} \mathrm{~N}^{-1}, 60 \mathrm{~kg} \mathrm{P}_{2} \mathrm{O}_{5}$ $\mathrm{ha}^{-1}$ y $110 \mathrm{~kg} \mathrm{~K}_{2} \mathrm{O} \mathrm{ha}{ }^{-1}$, en forma de nitrato amónico, ácido fosfórico y sulfato potásico, respectivamente.

Los resultados de rendimiento comercial acumulado indican que la dosis de riego afectó de forma significativa a los valores registrados en los meses de febrero, marzo, abril y mayo. Si bien entre la dosis $R_{2}$ y $R_{3}$ las diferencias no resultaron estadísticamente significativas.

Los restantes parámetros del cultivo (peso medio de los capítulos y componentes del destrío) resultaron poco afectados por la dosis de riego.

\section{Introducción}

El agua es uno de los factores de la producción más limitantes en los agrosistemas, siendo el riego de los cultivos el principal sector consumidor de agua. Por lo que ante la acusada escasez de este recurso natural, resulta imperiosamente necesario utilizar el agua de riego de la forma más eficiente posible.

Para establecer un programa racional de riego en alcachofa multiplicada por semilla, que cubra sus necesidades con las mínimas pérdidas posibles, es de primordial importancia conocer las necesidades de riego de este cultivo en las condiciones reales de los agrosistemas.

Ensayos previos realizados por este mismo grupo de trabajo, desarrollado en la finca experimental de Cajamar en Paiporta, con el cv. multiplicado por semilla Imperial Star en la campaña 2001-2002, en la que se compararon 3 dosis de riego, se obtuvieron los mejores resultados productivos con las dosis media y alta (5.472 y $7.265 \mathrm{~m}^{3} \mathrm{ha}^{-1}$, respectivamente) respecto a la dosis baja $\left(4.104 \mathrm{~m}^{3} \mathrm{ha}^{-1}\right.$ ) (Baixauli y Maroto 2011; Pomares et al., 2003). El 
objetivo de este ensayo fue evaluar los efectos de tres dosis de riego en el rendimiento y calidad comercial del cultivo de alcachofa de semilla en riego por goteo.

\section{Material y métodos}

La siembra del cv. Symphony (Nunhems) se realizó en semillero profesional sobre bandeja rellena de una mezcla de turba rubia y fibra de coco de 270 alvéolos el 8 de junio de 2010, siendo sometido a una pregerminación en cámara a una temperatura de $27^{\circ} \mathrm{C}$ y una humedad relativa del $90 \%$. Posteriormente las plántulas fueron repicadas a bandejas de 104 alvéolos cuando tenían entre 4 a 6 hojas verdaderas donde se desarrollaron hasta el momento del trasplante. La plantación se efectuó el 22/7/2010, con un marco de $1.67 \times 0.78$ $\mathrm{m}\left(0.77 \mathrm{pl} \mathrm{m}^{-2}\right)$ con sistema de riego localizado (por goteo). Posteriormente se efectuaron tres aplicaciones de ácido giberélico a $60 \mathrm{ppm}$ adicionando al caldo un abono foliar (Abofol) a una concentración de $1 \mathrm{gr} \mathrm{I}^{-1}$, realizando el primer tratamiento cuando las plantas tenían 6 y 10 hojas totalmente desarrolladas, en las fechas: 6/9/10, 21/9/10 y 6/10/10, respectivamente, con un gasto de caldo de unos $375 \mathrm{I} \mathrm{ha}^{-1}$. Para el cálculo de las dosis de riego se siguió el método propuesto por Doorenbos y Pruitt (1997), obteniendo la $\mathrm{ET}_{\mathrm{c}}$ a partir de la evapotranspiración de referencia $\left(\mathrm{ET}_{\mathrm{o}}\right.$ ), según el método Penman-Monteith (Allen et al., 1998) y los valores de coeficientes de cultivo $\left(\mathrm{K}_{\mathrm{c}}\right)$ de $0.35,1.20$ y 1.05 , durante los períodos inicial, intermedio y final, y una duración en días de los referidos períodos de 35 , 98, 119 y 28, para la fase inicial, de desarrollo, intermedia y final, respectivamente, aplicando los volúmenes de agua siguientes: 2.943, 4.305 y $6.031 \mathrm{~m}^{3} \mathrm{ha}^{-1}$, en los tratamientos $R_{1}, R_{2}, R_{3}$, respectivamente. La lluvia contabilizada durante el ciclo del cultivo fue de $374 \mathrm{~mm}$, equivalente a $3.740 \mathrm{~m}^{3} \mathrm{ha}^{-1}$. Estas dosis de riego resultan equivalentes al 68,100 y $140 \% E T_{c}$, para $R_{1}, R_{2}$ y $R_{3}$, respectivamente. Se aplicó el mismo abonado en las tres dosis de riego a base de $166 \mathrm{~kg} \mathrm{~N} \mathrm{ha}^{-1}, 60 \mathrm{~kg} \mathrm{P}_{2} \mathrm{O}_{5} \mathrm{ha}^{-1}$ y $110 \mathrm{~kg} \mathrm{~K}_{2} \mathrm{O}$ ha ${ }^{-1}$, en forma de nitrato amónico, ácido fosfórico y sulfato potásico respectivamente. Durante el cultivo en el período de invierno se produjeron varias heladas los días $4,16,17$ y 28 de diciembre de 2010 con $-2.5,-3,-1,-4$ y $-1^{\circ} \mathrm{C}$, respectivamente y los días $22,23,24$ y 25 de enero de 2011 , con $-4,-1.5,-2$ y $-1^{\circ} \mathrm{C}$, respectivamente, que afectó a los capítulos, al desarrollo de la planta $\mathrm{y}$ al rendimiento final, aunque por igual en todos los casos.

Se realizó un diseño estadístico de bloques al azar con 3 repeticiones, con 9 plantas por parcela elemental. Para el análisis estadístico se empleó el programa estadístico Stat. Graphics Plus.

Para cada una de las dosis de riego se contabilizó la producción comercial acumulada por meses durante el período productivo (desde el 4/11/10 al 17/5/11), el peso medio de sus capítulos, la producción de destrío como consecuencia de capítulos pequeños y deformes, de destrío por presencia de brácteas abiertas y el destrío total acumulado por meses. A lo largo del cultivo se hicieron 3 valoraciones del vigor de las plantas, la primera el 6 de septiembre, la segunda el 11 de noviembre de 2010 y la última el 15 de abril de 2011, adjudicándoles una puntuación desde 1 (para las hileras con muy poco vigor), hasta 5 (para aquellas en las que sus plantas fueron muy vigorosas).

\section{Resultados y discusión}

Respecto al rendimiento de los capítulos comerciales, los valores obtenidos con las dosis de riego media $\left(R_{2}\right)$ y alta $\left(R_{3}\right)$ fueron más altos para la producción acumulada total, que los obtenidos con la dosis baja $\left(R_{1}\right)$, registrándose diferencias significativas a nivel estadístico (d.s.n.e.) con una probabilidad $p<0.01$; en cambio, durante el periodo productivo desde febrero hasta mayo, no se apreciaron d.s.n.e. entre la dosis baja $\left(R_{1}\right)$ y la alta $\left(R_{3}\right)$ en la producción acumulada de febrero, marzo y abril, pero sí entre la dosis media $\left(R_{2}\right)$ y la baja $\left(R_{1}\right)$ para los meses de febrero, marzo y abril con una probabilidad $p<0.10, p<0.05$ y $p<0.01$, 
respectivamente. No obstante, entre las dosis $R_{2}$ y $R_{3}$ no se obtuvieron diferencias significativas en ninguno de los meses (tabla 1).

Tabla 1. Efecto de la dosis de riego en el rendimiento comercial acumulado mensualmente Rendimiento mensual comercial $\left(\mathrm{kg} \mathrm{m}^{-2}\right)$

\begin{tabular}{cccccccc}
\hline Tratamiento & Noviembre & Diciembre & Enero & Febrero & Marzo & Abril & Mayo \\
\hline R1 & 0.08 & 0.22 & 0.23 & $0.24 b$ & $0.25 \mathrm{~b}$ & $0.46 \mathrm{~B}$ & $1.06 \mathrm{~B}$ \\
\hline R2 & 0.18 & 0.36 & 0.37 & $0.38 a$ & $0.45 \mathrm{a}$ & $0.91 \mathrm{~A}$ & $1.62 \mathrm{~A}$ \\
\hline R3 & 0.13 & 0.28 & 0.30 & $0.31 a b$ & $0.35 \mathrm{ab}$ & $0.67 \mathrm{AB}$ & $1.68 \mathrm{~A}$ \\
\hline & n.s. & n.s. & n.s. & $\mathrm{p}<0.10$ & $\mathrm{p}<0.05$ & $\mathrm{p}<0.01$ & $\mathrm{p}<0.01$ \\
\hline
\end{tabular}

* Los valores medios seguidos de letras mayúsculas, minúsculas o minúsculas cursiva en cada columna indican diferencias significativas a $p<0.01, p<0.05$ y $p<0.10$, respectivamente. n.s. indica ausencia de diferencias significativas a $p<0.10$.

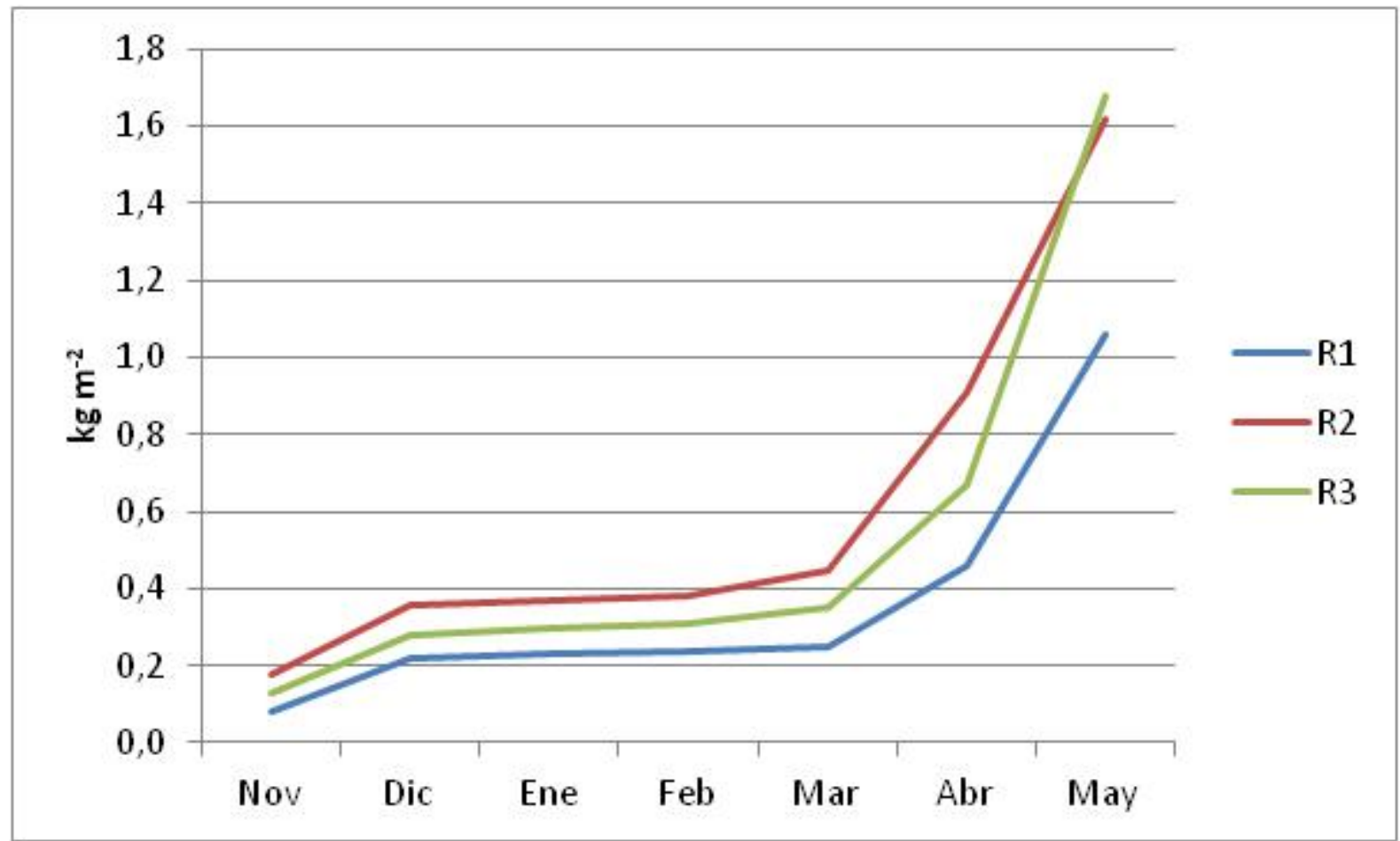

Figura 1. Evolución del rendimiento comercial de capítulos acumulado por meses durante el período productivo.

Asimismo, el peso medio mensual de los capítulos no resultó afectado por ninguna de las dosis de riego comparadas en ninguna de las observaciones (tabla 2). Estos resultados de rendimiento comercial y peso medio de sus capítulos, coinciden con los observados por este mismo grupo de trabajo, en un estudio realizado en la campaña 2001-02 con el cv. Imperial Star (Pomares et al., 2003).

Tabla 2. Efecto de la dosis de riego en el peso medio mensual de los capítulos Peso medio $\left(\mathrm{g}^{\text {capítulo }}{ }^{-1}\right)$

\begin{tabular}{cccccccc}
\hline Tratamiento & Noviembre & Diciembre & Enero & Febrero & Marzo & Abril & Mayo \\
\hline R1 & 127.3 & 111.3 & 120.0 & 127.5 & 150.0 & 181.3 & 138.6 \\
\hline R2 & 122.2 & 115.4 & 117.5 & 162.5 & 144.7 & 164.5 & 138.9 \\
\hline R3 & 134.9 & 119.6 & 114.2 & 116.7 & 162.2 & 178.0 & 148.0 \\
\hline & n.s. & n.s. & n.s. & n.s. & n.s. & n.s. & n.s. \\
\hline
\end{tabular}


* Los valores medios seguidos de letras mayúsculas, minúsculas o minúsculas cursiva en cada columna indican diferencias significativas a $p<0.01, p<0.05$ y $p<0.10$, respectivamente. n.s. indica ausencia de diferencias significativas a $p<0.10$.

En cuanto a la producción de destrío, únicamente se encontraron diferencias significativas en el rendimiento de destrío acumulado por capítulos pequeños y deformes correspondiente al mes abril (tabla 3 ) y en el rendimiento de capítulos abiertos correspondiente a mayo (tabla $4)$.

Tabla 3. Efecto de la dosis de riego en el destrío por capítulos pequeños y deformes acumulado mensualmente

\begin{tabular}{cccccccc}
\hline \multicolumn{7}{c}{ Rendimiento destrío por capítulos pequeños y deformes $\left(\mathrm{kg} \mathrm{m}^{-2}\right)$} \\
\hline Tratamiento & Noviembre & Diciembre & Enero & Febrero & Marzo & Abril & Mayo \\
\hline R1 & 0.01 & 0.01 & 0.03 & 0.03 & 0.04 & $0.13 a$ & 0.30 \\
\hline R2 & 0.00 & 0.00 & 0.09 & 0.01 & 0.01 & $0.02 b$ & 0.22 \\
\hline R3 & 0.00 & 0.00 & 0.00 & 0.00 & 0.00 & $0.00 b$ & 0.19 \\
\hline & n.s. & n.s. & n.s. & n.s. & n.s. & p $<0.10$ & n.s. \\
\hline
\end{tabular}

* Los valores medios seguidos de letras mayúsculas, minúsculas o minúsculas cursiva en cada columna indican diferencias significativas a $p<0.01, p<0.05$ y $p<0.10$, respectivamente. n.s. indica ausencia de diferencias significativas a $p<0.10$.

Tabla 4. Efecto de la dosis de riego en el destrío por capítulos abiertos acumulado mensualmente

\begin{tabular}{cccccccc}
\hline \multicolumn{7}{c}{ Rendimiento destrío por capítulos abiertos $\left(\mathrm{kg} \mathrm{m}^{-2}\right)$} \\
\hline Tratamiento & Noviembre & Diciembre & Enero & Febrero & Marzo & Abril & Mayo \\
\hline R1 & 0.01 & 0.01 & 0.03 & 0.03 & 0.05 & 0.07 & $0.17 \mathrm{~b}$ \\
\hline R2 & 0.00 & 0.02 & 0.04 & 0.04 & 0.04 & 0.04 & $0.25 a$ \\
\hline R3 & 0.00 & 0.01 & 0.02 & 0.02 & 0.02 & 0.02 & $0.13 \mathrm{~b}$ \\
\hline & n.s. & n.s. & n.s. & n.s. & n.s. & n.s. & $\mathrm{p}<0.10$ \\
\hline
\end{tabular}

${ }^{*}$ Los valores medios seguidos de letras mayúsculas, minúsculas o minúsculas cursiva en cada columna indican diferencias significativas a $p<0.01, p<0.05$ y $p<0.10$, respectivamente. n.s. indica ausencia de diferencias significativas a $p<0.10$.

Tabla 5. Efecto de la dosis de riego en el destrío total acumulado mensualmente Rendimiento destrío total $\left(\mathrm{kg} \mathrm{m}^{-2}\right)$

\begin{tabular}{cccccccc}
\hline Tratamiento & Noviembre & Diciembre & Enero & Febrero & Marzo & Abril & Mayo \\
\hline R1 & 0.02 & 0.02 & 0.06 & 0.10 & 0.13 & 0.24 & 0.51 \\
\hline R2 & 0.02 & 0.02 & 0.04 & 0.08 & 0.09 & 0.10 & 0.51 \\
\hline R3 & 0.00 & 0.01 & 0.02 & 0.10 & 0.10 & 0.11 & 0.41 \\
\hline & n.s. & n.s. & n.s. & n.s. & n.s. & n.s. & n.s. \\
\hline
\end{tabular}

* Los valores medios seguidos de letras mayúsculas, minúsculas o minúsculas cursiva en cada columna indican diferencias significativas a $p<0.01, p<0.05$ y $p<0.10$, respectivamente. n.s. indica ausencia de diferencias significativas a $p<0.10$.

Por otra parte, los datos de valoración del vigor de las plantas pusieron de manifiesto un marcado efecto de la dosis de riego en el desarrollo de las plantas, obteniéndose diferencias significativas en las tres valoraciones realizadas durante la fase de crecimiento vegetativo detectando un menor desarrollo de las plantas correspondientes a la dosis de riego más baja $\left(R_{1}\right)$ (tabla 6$)$. 
Tabla 6. Efecto de la dosis de riego en el vigor (escala de 0-5) de las plantas de alcachofa

\begin{tabular}{cccc}
\hline Tratamiento & $\begin{array}{c}\text { Vigor } \\
6 / 9 / 10\end{array}$ & $\begin{array}{c}\text { Vigor } \\
11 / 11 / 10\end{array}$ & $\begin{array}{c}\text { Vigor } \\
15 / 4 / 11\end{array}$ \\
\hline R1 & $1.08 \mathrm{~B}$ & $3.25 \mathrm{~b}$ & $3.83 \mathrm{~B}$ \\
\hline $\mathrm{R} 2$ & $2.33 \mathrm{~A}$ & $3.42 \mathrm{ab}$ & $4.83 \mathrm{~A}$ \\
\hline $\mathrm{R} 3$ & $2.75 \mathrm{~A}$ & $3.50 \mathrm{a}$ & $5.00 \mathrm{~A}$ \\
\hline & $\mathrm{p}<0.01$ & $\mathrm{p}<0.05$ & $\mathrm{p}<0.01$ \\
\hline
\end{tabular}

* Los valores medios seguidos de letras mayúsculas, minúsculas o minúsculas cursiva en cada columna indican diferencias significativas a $p<0.01, p<0.05$ y $p<0.10$, respectivamente. n.s. indica ausencia de diferencias significativas a $p<0.10$.

\section{Conclusiones}

Los resultados de rendimiento comercial acumulado indican que la dosis de riego afectó de forma significativa a los valores registrados en los meses de febrero, marzo, abril y mayo. Si bien entre la dosis media $\left(R_{2}\right)$ y alta $\left(R_{3}\right)$, las diferencias no resultaron estadísticamente significativas.

Los restantes parámetros del cultivo (peso medio de los capítulos y componentes del destrío) resultaron poco afectados por la dosis de riego.

La dosis de riego afectó de forma altamente significativa al vigor de la plantas, pero entre las dosis de riego media y alta, las diferencias no resultaron significativas.

\section{Bibliografía}

Allen, R.G., Pereira, L.S., Raes, D., Smith, M. (1998). Crop evapotranspiration: guidelines for computing crop water requirements. FAO Irrigation and Drainage. Paper 56. FAO, Roma.

Baixauli, C., Maroto, J. V. (2011). Cultivares de alcachofa propagable por semilla, respuesta al ácido giberélico. Nuevas variedades de reproducción por semilla y técnicas de producción en alcachofa. Ed. Académica Española, 258 pp.

Doorenbos, J., Pruitt, W.O. (1977). Las necesidades de agua de los cultivos. Estudio FAO. Riego y Drenaje 24. Roma, 194 pp.

Pomares, F., Baixauli, C., Aguilar, J.M., Giner, A., Tarazona, F., Estela, M. (2003). Memoria de Actividades 2002. Resultados de Ensayos Hortícolas. Efecto de diferentes dosis de riego en alcachofa de semilla. Fundación Ruralcaja Valencia, Generalitat Valenciana: pp 381. 